\title{
BOND PORTFOLIO'S DURATION AND INVESTMENT TERM-STRUCTURE MANAGEMENT PROBLEM
}

\author{
DAOBAI LIU
}

Received 30 November 2004; Revised 25 April 2005; Accepted 27 April 2005

In the considered bond market, there are $N$ zero-coupon bonds transacted continuously, which will mature at equally spaced dates. A duration of bond portfolios under stochastic interest rate model is introduced, which provides a measurement for the interest rate risk. Then we consider an optimal bond investment term-structure management problem using this duration as a performance index, and with the short-term interest rate process satisfying some stochastic differential equation. Under some technique conditions, an optimal bond portfolio process is obtained.

Copyright (c) 2006 Daobai Liu. This is an open access article distributed under the Creative Commons Attribution License, which permits unrestricted use, distribution, and reproduction in any medium, provided the original work is properly cited.

\section{Introduction}

Recent study shows that interest rate risk management is a crucial element of success in the bank industry (see [9], etc.). However, it is not very easy to accurately measure this risk.

Gap analysis (see [23], etc.) is the oldest approach to analyze investment portfolio risk, which was adapted from a kind of liquidity analysis that scheduled out projected cash flows generated from the portfolio. Combining fixed maturities and repricing distributions in this manner reflects a traditional gap report. The gap report seems to be a fairly complete reflection of interest rate risk, unfortunately, that is far from the case, especially for measuring the rate sensitive of embedded option securities.

The so-called weighted-average life is simply a measure that uses the cash weighted average of the time until the total cash flow is received. To make it clearer, let us consider a simple illustrative example. Suppose there are two bonds: the first bond will pay the holder \$100 two years later; the second bond will pay the holder \$50 one year later and $\$ 50$ two years later. The investor can get the same sum in the coming two years by holding these two bonds. For the first bond, the holder must wait two years to receive the money, but for the second bond, half of the total cash will be received one year later, and only half

Hindawi Publishing Corporation

Journal of Applied Mathematics and Stochastic Analysis

Volume 2006, Article ID 76920, Pages 1-19

DOI 10.1155/JAMSA/2006/76920 
of the total cash will be received two years later. Thus, for the first bond, the waiting time to get all cash is two years. But for the second bond, the weighted-average waiting time to get all cash (which is called the weighted-average life) is

$$
\frac{50}{100} \times 1+\frac{50}{100} \times 2=1.5 \text {. }
$$

The weighted-average life is not the best measure of rate sensitivity because it does not reflect the differences caused by the time value of money: bonds with earlier cash flows are more valuable than bonds with later cash flows. For example, the $\$ 50$ received one year later is more valuable than the $\$ 50$ received two years later usually.

It turns out that a weighted average of the present values of those cash flows is a better measurement of the bond's volatility. This average is called the bond's duration. For the above example, let the annual interest rate be $r>0$. Then the second bond's duration is

$$
\frac{50}{50+50 /(1+r)} \times 1+\frac{50 /(1+r)}{50+50 /(1+r)} \times 2=\frac{3+r}{2+r}<1.5
$$

In general, for a coupon bond with maturity $k$ (which will be called a $k$-coupon bond), the holder will receive coupon payments $C_{n}$ at time $n$, where $1 \leq n \leq k-1$, and will receive the last payment $C_{k}$ consists of the last coupon payment and the par value at maturity $k$. For such a coupon bond, similar to (1.1), the average waiting time to get the cash flow is

$$
\sum_{n=1}^{k} \frac{C_{n}}{\sum_{i=1}^{k} C_{i}} \times n .
$$

If the continuously compounded interest rate is $R$, then the weighted average of present values of the cash flows is

$$
\sum_{n=1}^{k} \frac{e^{-n R} C_{n}}{\sum_{i=1}^{k} e^{-i R} C_{i}} \times n:=D_{M} .
$$

We call $D_{M}$ the (traditional) duration (under continuously compounded interest rate) of this coupon bond (see $[2,3,7,9,22]$ ).

The (traditional) duration improves upon weighted-average life by reflecting the relative importance of the timing of the cash flows. It does this by weighting the time of receipt of each cash flow by the present value of the cash flow. The traditional duration has a very important property. Denote $P(R)$ to be the market value of this coupon bond, emphasizing the dependence on the interest rate $R$, then

$$
P(R):=\sum_{n=1}^{k} e^{-n R} C_{n} .
$$

We have

$$
\frac{d P}{d R}=\sum_{n=1}^{k}\left(-n e^{-n R} C_{n}\right)=-D_{M} P
$$


That is

$$
D_{M}=-\frac{1}{P} \frac{d P}{d R}
$$

This property characterizes the sensitivity of bond price to the interest rate. When the interest rate has a fluctuation $\Delta R$ instantaneously, by (1.7), we have

$$
\frac{P(R+\Delta R)-P(R)}{P(R)} \approx-D_{M} \Delta R .
$$

This means that if the $k$-coupon bond's duration is 10 , then every one basis point increase of the interest rate (i.e., $\Delta R=0.01 \%$ ) will result in ten basis point decrease of the bond price. In contrast, every one basis point decrease of the interest rate will result in ten basis point increase of the bond price. For example, for a coupon bond with duration 10 at time $t$ and with market value $100 \$$, if the interest rate increases $0.01 \%$, by (1.8), we have

$$
P(2.00 \%+0.01 \%) \approx P(2.00 \%)-P(2.00 \%) \times 10 \times 0.01 \%=100-0.1=99.9 .
$$

That is, the market value of this coupon bond will approximately decrease to $99.9 \$$; and if the interest rate decreases $0.01 \%$, the market value of this coupon bond will approximately increase to $100.1 \$$.

Due to this property, the traditional duration is a very important index which can reflect the interest rate risk. Unfortunately, there is a very uncomfortable defect of the traditional duration: it assumes that the yield curves are flat and shift in a parallel manner, the short-term yield is the same as the long-term yield. In (1.4), the discount interest rate for different waiting time is similar. That is, if the discount interest rate for one year it is $2.00 \%$, for ten years is also $2.00 \%$. Clearly, this is improper.

To get rid of the above-mentioned defect, we could modify (1.4) by using different discount interest rate for different waiting time. That is,

$$
\bar{D}_{M}:=\sum_{n=1}^{k} \frac{e^{-n R_{n}} C_{n}}{\sum_{i=1}^{k} e^{-i R_{i}} C_{i}} \times n .
$$

However, we know that the traditional duration can be used to characterize interest rate risk directly, since it has an important property (1.7). But the "modified duration" $\bar{D}_{M}$ defined by (1.10) does not have such a property. Thus, to find a proper index which not only can offset the defect of the traditional duration but also can characterize interest rate risk, the modification like (1.10) is insufficient. There are several definitions about modified duration, such as key rate duration (see [14]) and efficient duration (see [9, Chapter 4], and for other definitions of "modified duration"). But still can not get rid of the defects.

In what follows, we will introduce a more general definition of duration (which might be regarded as an index of interest rate risk). We will work under a stochastic short-term interest rate model.

As we know, the coupon bond can be viewed as a portfolio of zero-coupon bonds. Hence, we need only to consider the portfolio of zero-coupon bonds below. 
We assume that, at any given time, there are always $N$ zero-coupon bonds being traded continuously in the bond market. At time 0 (the initial time), the maturities of these $N$ bonds are $k=1,2, \ldots, N$, respectively. That is, after every unit time, there will be a bond matured. We call the zero-coupon bond maturing at time $k$ a $k$-bond. At time $t=1$, the 1 bond is matured, and at the same time, a new bond with maturity $N+1$ is issued. Similar, at any time $t \geq 0$, the maturities of these $N$ bonds are $k=[t]+1,[t]+2, \ldots,[t]+N$, where $[t]$ is the largest integer less than or equal to $t$. Let the price of $k$-bond at time $t$ be $P(t, k)$. For convenience, we assume that $P(k, k)=1, k=1,2, \ldots$ Denote

$$
f(t, k):=\frac{1}{k-t} \ln \frac{P(k, k)}{P(t, k)}=-\frac{\ln P(t, k)}{k-t}
$$

to be the continuously compounded yield of $k$-bond at time $t$. Thus

$$
P(t, k)=e^{-f(t, k)(k-t)}, \quad t \in[0, k] .
$$

The relationship between the yields to maturity of zero-coupon bonds and the terms to maturity is known as the terms structure of interest rates, and it is represented graphically by the yield curve. In the definition of traditional duration, it assumes that the yield curves are flat and shift in a parallel manner. In fact, the term structure exhibits different patterns of changes over time. The yield curve can be of any of the following four shapes (for more details, see [12]).

(1) The normal yield curve, that is, the yield curve upward sloping: the short-term yield is lower than the long-term yield.

(2) Inverted yield curve: the short-term yield is higher than the long-term yield.

(3) Flat yield curve: the short-term yield is the same as the long-term yield.

(4) Humped yield curve: the intermediate yield is higher than both the short-term and long-term yields.

Theoretical models of the term structure of interest rates are of interest to both practitioners and financial academics. In this paper, we will use a continuous time diffusion model to characterize the dynamics of the short-term interest rate. Denote $r(\cdot)$ to be the short-term interest rate process, which satisfies some stochastic differential equation. Under this stochastic short-term interest rate model, we will proposes a new interest rate risk measure that would help investors and bank monitors in assessing interest rate risk.

We know that the price of $k$-bond at time $t$ is determined by interest rate $r(t)$ under some technique conditions. That is, there are smooth functions $\varphi^{k}(\cdot, \cdot)$ such that

$$
P(t, k)=\varphi^{k}(t, r(t)), \quad k=1,2,3, \ldots
$$

Denote $V(t, r(t))$ to be the investor's total wealth invested in zero-coupon bonds at time $t$, corresponding to bond portfolio $\pi(t):=\left(\pi_{1}(t), \pi_{2}(t), \ldots, \pi_{N}(t)\right)$, where $\pi_{n}(t)$ is the share number of the $([t]+n)$-bond held by investor at time $t$ :

$$
V(t, r(t))=\sum_{n=1}^{N} \pi_{n}(t) P(t,[t]+n)=\sum_{n=1}^{N} \pi_{n}(t) \varphi^{[t]+n}(t, r(t)) .
$$


Inspired by (1.7), the duration of bond portfolio $\pi(t)$ under stochastic interest rate model should be defined as follows.

Definition 1.1. For given time $t$ and bond portfolio $\pi(t)$, denote

$$
D(t, \pi(t)):=-\frac{V_{r}(t, r(t))}{V(t, r(t))} .
$$

Call $D(t, \pi(t))$ the duration of bond portfolio $\pi(t)$.

Similar to the traditional duration of coupon bond, zero-coupon bond portfolio's duration $D(t ; \pi(t))$ defined by (1.15) is also a very good index of interest rate risk of bond portfolio $\pi(t)$. That is, if bond portfolio's duration equals 10, then for every one basis point increase of short-term interest rate at time $t$, there will be ten basis point decrease of market value of the bond portfolio $\pi(t)$. Contrarily, for every one basis point decrease of short-term interest rate at time $t$, there will be ten basis point increase of market value of the bond portfolio $\pi(t)$. To compare with the traditional duration, we need not assume that the term structure is flat. Moreover, the traditional duration is a special case of Definition 1.1. When $r(t) \equiv R$, we have $\varphi^{k}(t, R)=e^{-R(k-t)}$. Then if $t=0, N=k$, and $\pi_{n}(0)=C_{n}, n=1,2, \ldots, N$, by Definition 1.1 and (1.13), we have

$$
D(0, C(t))=\sum_{n=1}^{k} \frac{C_{n} e^{-n R}}{V(0, R)} \times n,
$$

where

$$
V(0, R)=\sum_{n=1}^{k} C_{n} e^{-n R} .
$$

This time, the bond portfolio's duration $D(0, C(t))$ is similar to the traditional duration defined in (1.4).

Let $\Pi_{\mathrm{ad}}[0, T]$ be the set of admissible bond portfolio processes (see Section 3 for details). Denote $x$ and $X(T, \pi(t))$ to be initial wealth and the total wealth at time $T$, respectively. Moreover,

$$
E[R(T, \pi(t))]:=\frac{1}{T} \ln \frac{X(T, \pi(t))}{x}, \quad \forall \pi(\cdot) \in \Pi_{\mathrm{ad}}[0, T]
$$

is the investment yield corresponding to admissible portfolio process $\pi(\cdot) \in \Pi_{\mathrm{ad}}[0, T]$. Then, under the above index of bond portfolio's interest rate risk, the bonds investment term-structure management problem can be stated as follows.

Problem 1.2. For given initial wealth $x>0$, find an admissible bond portfolio process $\bar{\pi}(\cdot) \in \Pi_{\mathrm{ad}}[0, T]$ such that

$$
\begin{gathered}
|D(t ; \bar{\pi}(t))| \leq D_{0}, \quad \text { a.s., a.e. } t \in[0, T], \\
E[R(T, \bar{\pi}(t))]=\sup _{\pi(\cdot) \in \Pi_{\mathrm{ad}}[0, T]}\left\{E[R(T, \pi(t))]|| D(t ; \pi(t)) \mid \leq D_{0} \text {, a.s., a.e. } t \in[0, T]\right\} .
\end{gathered}
$$


The rest of this paper is organized as follows. In Section 2, we will give a definition of bond portfolio's duration under stochastic interest rate model. In Section 3, we will formulate the bond investment term structure management problem and the optimal portfolio process will be characterized. Some special case will be discussed in Section 4 .

\section{Continuous-time bond market and the duration of zero-coupon bond's portfolio}

In this section, we will consider the duration of zero-coupon bond's portfolio under a given stochastic interest rate model.

Suppose that the short-term interest rate (or the instantaneous interest rate) $r(\cdot)$ is a diffusion process which satisfies the following stochastic differential equation:

$$
d r(t)=\eta(t, r(t)) d t+\zeta(t, r(t)) d W(t), \quad t \geq 0, r(0)=r_{0}
$$

Here $\{W(t), t \geq 0\}$ is a standard Brownian motion, defined on a complete filtered probability space $\left(\Omega, \mathscr{F}_{\mathbb{F}}, \mathbb{P},\left\{\mathscr{F}_{t}\right\}_{t \geq 0}\right)$, and $\mathscr{F}_{t}$ is the $\mathbb{P}$-augmentation of the filtration $\mathscr{F}_{t}^{W}=$ $\sigma(W(s) ; 0 \leq s \leq t)$ generated by $W(\cdot)$. The coefficients $\eta(\cdot, \cdot)$ and $\zeta(\cdot, \cdot):[0, T] \times \mathbb{R} \rightarrow \mathbb{R}$ are some continuous functions.

As mentioned in the previous section, we assume that there are always one common bond and $N$ zero-coupon bonds transacted continuously in the bond market. At any time $t \geq 0$, the maturities of these $N$ bonds are $k=[t]+1,[t]+2, \ldots,[t]+N$. Denote the price of $k$-bond and common bond at time $t$ to be $P(t, k)$ and $P_{0}(t)$, respectively. The price process of common bond $P_{0}(\cdot)$ satisfies the following differential equation:

$$
d P_{0}(t)=P_{0}(t) r(t) d t, \quad t \geq 0, P_{0}(0)=1
$$

We usually take the common bond as an account of money market (or bank).

The price processes of $k$-bond, $P(\cdot, k), k=1,2, \ldots$, satisfy the following stochastic differential equations:

$$
\begin{gathered}
d P(t, k)=[P(t, k) r(t)+\Lambda(t, r(t)) Z(t, k)] d t+Z(t, k) d W(t), \quad t \in[(k-N) \vee 0, k), \\
P(k, k)=1,
\end{gathered}
$$

where the extra component $Z(\cdot, k)$ is uniquely determined, and $\Lambda(t, r(t))$ is the $k$-bond's market price of risk.

Equation (2.3) is called a backward stochastic differential equation (BSDE, for short), and $\{(P(\cdot, k), Z(\cdot, k)), 1 \leq k \leq N\}$ is called an adapted solution to (2.3). For the existence and uniqueness of adapted solution to BSDE (2.3), some assumptions for the coefficient in (2.1) and (2.3) are required. The following conditions are sufficient but not necessary.

(H) Functions $\eta, \zeta$, and $\Lambda$ are bounded and smooth. Moreover, $\eta_{r}$ and $\zeta_{r}$ are bounded.

LEMMA 2.1. Under the assumption $(H)$, there is a unique adapted solution $(P(\cdot, k), Z(\cdot, k))$ of $\operatorname{BSDE}(2.3)$, and for any given $k=1,2, \ldots$,

$$
P(t, k)>0, \quad \text { a.s. , a.e. } t \in[(k-N) \vee 0, k] \text {. }
$$


Moreover, there is a unique classical solution $\varphi^{k}$ of the following partial differential equation:

$$
\begin{gathered}
\varphi_{t}^{k}(t, r)+(\eta(t, r)-\Lambda(t, r) \zeta(t, r)) \varphi_{r}^{k}(t, r)+\frac{1}{2} \zeta(t, r)^{2} \varphi_{r r}^{k}(t, r)-r \varphi^{k}(t, r)=0, \\
(t, r) \in[0, k) \times \mathbb{R}, \\
\varphi^{k}(k, r)=1, \quad r \in \mathbb{R},
\end{gathered}
$$

such that

$$
\begin{gathered}
P(t, k)=\varphi^{k}(t, r(t)), \quad \text { a.s., a.e. } t \in[(k-N) \vee 0, k), \\
Z(t, k)=\zeta(t, r(t)) \varphi_{r}^{k}(t, r(t)), \quad \text { a.s., a.e. } t \in[(k-N) \vee 0, k) .
\end{gathered}
$$

Proof. First of all, similar to the results of Ma and Yong [15, Theorem 2.2 in Chapter 4], there is a unique classical solution of partial differential equation (2.5). Moreover, functions $\varphi_{t}^{k}, \varphi_{r}^{k}$, and $\varphi_{r r}^{k}$ are bounded. Consequently, we can check that $(P(\cdot, k), Z(\cdot, k))$, defined by (2.6) and (2.7), is an adapted solution of backward stochastic differential equation (2.3). On the other hand, by the results in [16], we know that the adapted solution of $\operatorname{BSDE}(2.3)$ is unique. Now we let

$$
\begin{gathered}
\theta(T)=e^{-\int_{0}^{T} \lambda(t, r(t)) d W(t)-(1 / 2) \int_{0}^{T}|\lambda(t, r(t))|^{2} d t} \\
W^{\lambda}(t)=W(t)+\int_{0}^{t} \lambda(s, r(s)) d s, \quad t \in[0, T] .
\end{gathered}
$$

Denote $\mathbb{P}^{\lambda}$ to be a probability measure such that $d \mathbb{P}^{\lambda} / d \mathbb{P}=\theta(T)$. Then it is equivalent to $\mathbb{P}$. By Girsanov's theorem, $W^{\lambda}(\cdot)$ is a standard Brownian motion under $\mathbb{P}^{\lambda}$. By $(2.3)$, we have

$$
d P(t, k)=P(t, k) r(t) d t+Z(t, k) d W^{\lambda}(t), \quad P(k, k)=1 .
$$

Consequently,

$$
\begin{aligned}
P(t, k) & =E^{\lambda}\left[e^{-\int_{t}^{k} r(s) d s}-\int_{t}^{k} e^{-\int_{t}^{s} r(\tau) d \tau} Z(\tau, k) d W(\tau) \mid \mathscr{F}_{t}\right] \\
& =E^{\lambda}\left[e^{-\int_{t}^{k} r(s) d s} \mid \mathscr{F}_{t}\right],
\end{aligned}
$$

where $E^{\lambda}$ is the expectation under the probability measure $\mathbb{P}^{\lambda}$. Then (2.4) holds.

Denote

$$
z(t, k)=\frac{Z(t, k)}{P(t, k)}, \quad t \in[(k-N) \vee 0, k]
$$

By (2.7), we have

$$
z(t, k)=\zeta(t, r(t)) \frac{\varphi_{r}^{k}(t, r(t))}{\varphi^{k}(t, r(t))}, \quad \text { a.s., a.e. } t \in[(k-N) \vee 0, k)
$$


Now, we consider an investor who has initial wealth $x>0$. Denote $B(t)$ and $\pi_{n}(t)$ to be the market value of common bond and share number of $([t]+n)$-bond held by the investor at time $t$, respectively, where $n=1,2, \ldots, N$. We call $(B(\cdot), \pi(\cdot))$ an investment strategy. For given trading strategy $(B(\cdot), \pi(\cdot))$, denote the total wealth of the investor at time $t$ by $X(t)$,

$$
X(t):=B(t)+\sum_{n=1}^{N} \pi_{n}(t) P(t,[t]+n), \quad t \in[0, T] .
$$

It is clear that $X(0)=x$.

Definition 2.2. For a given investment term $T>0$, the investment strategy $(B(\cdot), \pi(\cdot))$ is said to be self-financing on $[0, T]$, if

$$
d X(t)=B(t) r(t) d t+\sum_{n=1}^{N} \pi_{n}(t) d P(t,[t]+n), \quad \text { a.s., a.e. } t \in[0, T] .
$$

It is easy to check that the wealth process $X(\cdot)$ corresponding to self-financing investment strategy $(B(\cdot), \pi(\cdot))$ satisfies the following stochastic differential equation:

$$
\begin{gathered}
d X(t)=\left\{X(t) r(t)+\sum_{n=1}^{N} \pi_{n}(t) \Lambda(t, r(t)) P(t,[t]+n) z(t,[t]+n)\right\} d t \\
+\sum_{n=1}^{N}\left\{\pi_{n}(t) P(t,[t]+n) z(t,[t]+n)\right\} d W(t), \quad t \geq 0, \\
X(0)=x .
\end{gathered}
$$

Hereafter, we always assume that the investment strategy $(B(\cdot), \pi(\cdot))$ is self-financing. By (2.15), the wealth process $X(\cdot)$ can be determined by $\pi(\cdot)=\left(\pi_{1}(\cdot), \pi_{2}(\cdot), \ldots, \pi_{N}(\cdot)\right)$ and initial wealth $x$. Define

$$
\begin{aligned}
\Pi[0, T]:= & \left\{\pi(\cdot)=\left(\pi_{1}(\cdot), \pi_{2}(\cdot), \ldots, \pi_{N}(\cdot)\right) \mid \pi(\cdot):[0, T] \times \Omega \longrightarrow \mathbb{R}^{N},\right. \\
& \text { is } \left.\left\{\mathscr{F}_{t}\right\}_{t \geq 0} \text {-adapted process, satisfying } E \int_{0}^{T}|\pi(t)|^{2} d t<\infty\right\} .
\end{aligned}
$$

Any $\pi(\cdot) \in \Pi[0, T]$ is called a bond portfolio process.

To emphasize the dependence of portfolio process $\pi(\cdot) \in \Pi[0, T]$ and initial wealth $x$, we denote the investor's total wealth at time $t$ to be $X(t, x, \pi(\cdot))$, and the market value of common bond held by the investor to be $B(t, x, \pi(\cdot))$. Thus

$$
B(t, x, \pi(\cdot)):=X(t, x, \pi(\cdot))-\sum_{n=1}^{N} \pi_{n}(t) P(t,[t]+n) .
$$

By (2.6) and (2.17), we have

$$
X(t, x, \pi(\cdot))=B(t, x, \pi(\cdot))+\sum_{n=1}^{N} \pi_{n}(t) \varphi^{[t]+n}(t, r(t)) .
$$


For given portfolio process $\pi(\cdot) \in \Pi[0, T]$ and initial wealth $x$, this equation gives the relationship between investor's total wealth and short-term interest rate at time $t$. Hence, we will write $X(t, x, \pi(\cdot))$ as $X(t, r(t) ; x, \pi(\cdot))$ sometimes. Similar to the definition of zerocoupon bond portfolio's duration, the duration at time $t$ of bond portfolio process $\pi(\cdot) \in$ $\Pi[0, T]$ is defined as following.

Definition 2.3. For a given $t \in[0, T]$, if $X(t, r(t) ; x, \pi(\cdot)) \neq 0$, a.s., let

$$
D(t ; \pi(t)):=-\frac{1}{X(t, r(t) ; x, \pi(\cdot))} \frac{\partial X(t, r(t) ; x, \pi(\cdot))}{\partial r} .
$$

Call $D(t ; \pi(t))$ the duration of bond portfolio process $\pi(\cdot) \in \Pi[0, T]$ at time $t$.

If $X(t) \neq 0$, a.s., a.e. $t \in[0, T]$, we can denote

$$
\alpha_{n}(t):=\frac{\pi_{n}(t) P(t,[t]+n)}{X(t)}, \quad n=1,2, \ldots, N
$$

to be the proportion of $([t]+n)$-bond's market value held by the investor to his total wealth at time $t$. Consequently, we have

$$
\begin{aligned}
D(t ; \pi(\cdot)) & =-\frac{1}{X(t)} \sum_{n=1}^{N} \pi_{n}(t) \varphi_{r}^{[t]+n}(t, r(t))=-\sum_{n=1}^{N} \frac{\pi_{n}(t)}{X(t)} \varphi_{r}^{[t]+n}(t, r(t)) \\
& =-\sum_{n=1}^{N} \frac{\pi_{n}(t) \varphi^{[t]+n}(t, r(t))}{X(t)} \frac{\varphi_{r}^{[t]+n}(t, r(t))}{\varphi^{[t]+n}(t, r(t))} \\
& =-\sum_{n=1}^{N} \alpha_{n}(t) \frac{\varphi_{r}^{[t]+n}(t, r(t))}{\varphi^{[t]+n}(t, r(t))}=-\sum_{n=1}^{N} \alpha_{n}(t) \frac{z(t,[t]+n)}{\zeta(t, r(t))} .
\end{aligned}
$$

The last equation is derived from (2.11). Take $\bar{D}(t ; \alpha(\cdot))$ instead of $D(t ; \pi(t))$, then

$$
\begin{gathered}
\bar{D}(t ; \alpha(\cdot))=-\sum_{n=1}^{N} \alpha_{n}(t) \frac{\varphi_{r}^{[t]+n}(t, r(t))}{\varphi^{[t]+n}(t, r(t))}, \\
\bar{D}(t ; \alpha(\cdot))=-\sum_{n=1}^{N} \alpha_{n}(t) \frac{z(t,[t]+n)}{\zeta(t, r(t))} .
\end{gathered}
$$

The relationship between $\bar{D}(t ; \alpha(\cdot))$ and $D(t ; \pi(t))$ is

$$
\bar{D}(t ; \alpha(\cdot))=D(t ; X(t) \alpha(t))
$$

\section{Bond investment term-structure management problem}

For a given investment term $T$, suppose there is an investor who wants to invest his initial wealth $x>0$ in the market. Then the term-structure management problem he will face is the following: at any time $t \in[0, T]$, he must determine the term structure of his portfolio, that is, how much money will be invested in different bonds. In other words, 
the investment problem is to select an appropriate portfolio process $\pi(\cdot) \in \Pi[0, T]$ which must satisfy some additional constraints, to achieve the investor's objective: return, risk, and liquidity.

Now we introduce the constraint conditions and the investor's objective. Firstly, the investor must guarantee that he will never be bankrupt during $[0, T]$. That is, the investor's wealth process $X(\cdot)$ must satisfies the following constraint:

$$
X(t)>0, \quad \text { a.s., a.e. } t \in[0, T] \text {. }
$$

As mentioned in Section 2, we denote $\alpha_{i}(t), i=1,2, \ldots, N$, to be the ratio at time $t$ of the market value of $([t]+i)$-bond held by the investor to his total wealth, that is, $\pi_{i}(t)=$ $\alpha_{i}(t) X(t)$. Now we denote

$$
\mathscr{A}[0, T]:=\left\{\alpha(\cdot):[0, T] \times \Omega \longrightarrow \mathbb{R}^{N} \mid \alpha(\cdot) \text { is }\left\{\mathscr{F}_{t}\right\}_{0 \leq t \leq T} \text {-adapted bounded process }\right\}
$$

to be the feasible bond portfolio process set on $[0, T]$. Any $\alpha(\cdot) \in \mathscr{A}[0, T]$ is called a feasible bond portfolio process, or portfolio process, for short.

For any self-financing portfolio process $\alpha(\cdot) \in \mathscr{A}[0, T]$ and initial wealth $x$, it is easy to check that the investor's total wealth process $X(\cdot)$ satisfies the following stochastic differential equation:

$$
\begin{aligned}
d X(t)=X(t)\left\{\left[r(t)+\sum_{n=1}^{N} \alpha_{n}(t) \Lambda(t, r(t)) z(t,[t]+n)\right] d t\right. & \\
& \left.+\sum_{n=1}^{N} \alpha_{n}(t) z(t,[t]+n) d W(t)\right\}, \quad t \geq 0, \quad X(0)=x .
\end{aligned}
$$

Similar to Section 2, sometimes we will write the investor's total wealth at time $t$ to be $X(t, x, \alpha(\cdot))$, emphasizing the dependent of initial wealth $x$ and the portfolio process $\alpha(\cdot) \in \mathscr{A}[0, T]$. By stochastic differential equation (3.3), we have

$$
X(t, x, \alpha(\cdot))=x e^{\int_{0}^{t}\left[r(t)+\Lambda(t, r(t)) h(t, \alpha(t))-(1 / 2) h(t, \alpha(t))^{2}\right] d t+\int_{0}^{t} h(t, \alpha(t)) d W(t)},
$$

where

$$
h(t, \alpha(t)):=\sum_{n=1}^{N} \alpha_{n}(t) z(t,[t]+n) .
$$

Hence, when $x>0$, we have

$$
X(t, x, \alpha(\cdot))>0, \quad \text { a.s., a.e. } t \geq 0 \text {. }
$$

Secondly, interest rate risk must be considered by the investor. We know that the market value of bond portfolio will be influenced by the movement of interest rate. Thus the investor wants to know that if there is one basis point change of short-term interest rate, how many basis points the market value of his portfolio will be changed? Usually, there is 
an interest rate risk limit which can be accepted by the investor. So we assume that there is a constant $D_{0} \geq 0$ such that the admissible bond portfolio process $\alpha(\cdot)$ must satisfy the following constraint:

$$
|\bar{D}(t ; \alpha(\cdot))| \leq D_{0}, \quad \text { a.s., a.e. } t \in[0, T],
$$

where $\bar{D}(t ; \alpha(\cdot)):=D(t ; \alpha(t) X(t, x, \alpha(\cdot)))$. Inequality (3.7) is called the investor's interest rate risk constraint. It means that, for one basis point instantaneous change of the shortterm interest rate at any time $t \in[0, T]$, the change of bond portfolio's market value accepted by investor will be no more than $D_{0}$ basis point.

Thirdly, the admissible bond portfolio will satisfy some constraint conditions. For example, there may be short sale constraint or loan constraint and so on. In this paper, we assume that there is a closed convex set $\Gamma \subset \mathbb{R}^{n}, 0 \in \Gamma$, such that the admissible bond portfolio processes must satisfy the following constraint conditions:

$$
\alpha(t) \in \Gamma, \quad \text { a.s., a.e. } t \in[0, T] .
$$

So we denote

$$
\begin{aligned}
\mathscr{A}_{\mathrm{ad}}[0, T]:= & \left\{\alpha(\cdot)=\left(\alpha_{1}(\cdot), \alpha_{2}(\cdot), \ldots, \alpha_{N}(\cdot)\right) \mid \alpha(\cdot):[0, T] \times \Omega \longrightarrow \Gamma,\right. \\
& \text { is bounded } \left.\left\{\mathscr{F}_{t}\right\}_{0 \leq t \leq T} \text {-adapted process }\right\}
\end{aligned}
$$

to be the admissible bond portfolio processes set. Any $\alpha(\cdot) \in \mathscr{A}_{\mathrm{ad}}[0, T]$ is called an admissible bond portfolio process.

Remark 3.1. There are some constraint set in real markets. The first example is

$$
\Gamma:=\left\{\alpha=\left(\alpha_{1}, \alpha_{2}, \ldots, \alpha_{N}\right) \in \mathbb{R}^{N} \mid a_{n} \geq 0, n=1,2, \ldots, N\right\},
$$

which means that there is a short sale constraint in bond market. The second example is, for a given constant $\alpha_{0} \in \mathbb{R}$,

$$
\Gamma:=\left\{\alpha=\left(\alpha_{1}, \alpha_{2}, \ldots, \alpha_{N}\right) \in \mathbb{R}^{N} \mid \sum_{n=1}^{N} \alpha_{n} \leq 1-\alpha_{0}\right\} .
$$

If $\alpha_{0}=0$, it means that there is loan constraint in market. In fact, if we denote $B(t, x, \alpha(\cdot))$ to be the amount of money in bank account owned by the investor at time $t$, then $\alpha(\cdot) \in$ $\mathscr{A}_{\mathrm{ad}}[0, T]$ means that

$$
B(t, x, \alpha(\cdot))=X(t, x, \alpha(\cdot))\left(1-\sum_{n=1}^{N} \alpha_{n}\right) \geq \alpha_{0} X(t, x, \alpha(\cdot))=0 .
$$

If the investor wants to guarantee certain liquidity of his portfolio, then he has $\alpha_{0}>0$. That is, the proportion of the current deposit he has to hold in bank account to his total wealth will be no less than $\alpha_{0}>0$. Thus, we have

$$
\frac{B(t, x, \alpha(\cdot))}{X(t, x, \alpha(\cdot))}=1-\sum_{n=1}^{N} \alpha_{n} \geq \alpha_{0}
$$


Corresponding to the case $\alpha_{0}>0$, we call (3.8) a liquidity constraint. The case $\alpha_{0}<0$ can be regarded as the case where the ratio of the amount of money that he can borrow from bank account to his total wealth will be no more than $-\alpha_{0}$. The third example is, for given $\alpha_{0}>0$,

$$
\Gamma:=\left\{\alpha=\left(\alpha_{1}, \alpha_{2}, \ldots, \alpha_{N}\right) \in \mathbb{R}^{N} \mid a_{n} \geq 0, n=1,2, \ldots, N, \sum_{n=1}^{N} \alpha_{n} \leq 1-\alpha_{0}\right\} .
$$

For an initial wealth $x$ and an admissible bond portfolio processes $\alpha(\cdot) \in \mathscr{A}_{\mathrm{ad}}[0, T]$, the total wealth of the investor at time $T$ is $X(T, x, \alpha(\cdot))$. Denote

$$
R(T, x, \alpha(\cdot)):=\frac{1}{T} \ln \frac{X(T, x, \alpha(\cdot))}{x} .
$$

We call $R(T, x, \alpha(\cdot))$ the investment yield (or investment return rate) on $[0, T]$. By (3.4), we have

$$
R(T ; x, \alpha(\cdot))=\frac{1}{T}\left[\int_{0}^{T} r(t)+\Lambda(t, r(t)) h(t, \alpha(t))-\frac{1}{2} h(t, \alpha(t))^{2} d t+\int_{0}^{T} h(t, \alpha(t)) d w(t)\right] .
$$

Thus, the investment yield does not depend on the initial wealth $x$. So we denote $R(T$, $\alpha(\cdot))$ to be the yield on $[0, T]$, corresponding to the admissible bond portfolio processes $\alpha(\cdot) \in \mathscr{A}_{\mathrm{ad}}[0, T]$, instead of $R(T, x, \alpha(\cdot))$.

Finally, we assume that the investor's objective is to find an admissible bond portfolio processes $\alpha(\cdot) \in \mathscr{A}_{\mathrm{ad}}[0, T]$ maximizing the expected investment yield (or investment return rate) $E[R(T, \alpha(\cdot))]$.

Now we formulate the bond investment term-structure management problem.

Problem 3.2. For given initial wealth $x>0$, select an admissible bond portfolio process $\alpha^{*}(\cdot) \in \mathscr{A}_{\mathrm{ad}}[0, T]$, which satisfies the duration constraint condition (3.7), to maximize the expected return rate $E[R(T, \alpha(\cdot))]$. That is,

$$
E\left[R\left(T, \alpha^{*}(\cdot)\right)\right]=\sup _{\alpha(\cdot) \in \mathscr{A}_{\mathrm{ad}}[0, T]}\left\{E[R(T, \alpha(\cdot))]|| \bar{D}(t ; \alpha(\cdot)) \mid \leq D_{0} \text {, a.s., a.e. } t \in[0, T]\right\} .
$$

\section{Denote}

$$
\overline{\mathscr{A}}_{\mathrm{ad}}[0, T]:=\left\{\alpha(\cdot) \in \mathscr{A}_{\mathrm{ad}}[0, T] \mid \bar{D}(t ; \alpha(\cdot)) \leq D_{0} \text {, a.s., a.e. } t \in[0, T]\right\},
$$

and let

$$
U(T):=\sup _{\alpha(\cdot) \in \bar{A}_{\mathrm{ad}}[0, T]} E[R(T ; \alpha(\cdot))] .
$$

Any portfolio process $\alpha^{*}(\cdot) \in \overline{\mathscr{A}}_{\mathrm{ad}}[0, T]$ which satisfies

$$
E\left[R\left(T ; \alpha^{*}(\cdot)\right)\right]=U(T)
$$

is called an optimal portfolio process of term-structure management Problem 3.2. 
Lemma 3.3. The duration constraint condition (3.7) is equivalent to

$$
\left|\sum_{n=1}^{N} \alpha_{n}(t) \frac{\varphi_{r}^{[t]+n}(t, r(t))}{\varphi^{[t]+n}(t, r(t))}\right| \leq D_{0}, \quad \text { a.s., a.e. } t \in[0, T],
$$

which is also equivalent to

$$
\left|\sum_{n=1}^{N} \alpha_{n}(t) z(t,[t]+n)\right| \leq D_{0} \zeta(t, r(t)), \quad \text { a.s., a.e. } t \in[0, T] .
$$

Proof. By (2.23), we have

$$
\bar{D}(t ; \alpha(\cdot))=-\sum_{n=1}^{N} \alpha_{n}(t) \frac{z(t,[t]+n)}{\zeta(t, r(t))} .
$$

Then the duration constraint condition (3.7) is equivalent to the following:

$$
\left|\sum_{n=1}^{N} \alpha_{n}(t) z(t,[t]+n)\right| \leq D_{0} \zeta(t, r(t)), \quad \text { a.s., a.e. } t \in[0, T] \text {. }
$$

Clearly, (3.21) is similar.

By (3.22), for a given $t \in[0, T]$, denote

$$
\begin{array}{r}
\Gamma(t):=\left\{\alpha: \Omega \longrightarrow \Gamma \mid \alpha \text { is } \mathscr{F}_{t}\right. \text {-measurable, bounded, } \\
\left.\left|\sum_{n=1}^{N} \alpha_{n} z(t,[t]+n)\right| \leq D_{0} \zeta(t, r(t)), \text { a.s. }\right\} .
\end{array}
$$

Theorem 3.4. Process $\alpha^{*}(\cdot) \in \overline{\mathscr{A}}_{\mathrm{ad}}[0, T]$ is an optimal bond portfolio process of termstructure management Problem 3.2, if and only if

$$
\begin{aligned}
& \left|\Lambda(t, r(t))-\sum_{n=1}^{N} \alpha_{n}^{*}(t) z(t,[t]+n)\right| \\
& \quad \leq\left|\Lambda(t, r(t))-\sum_{n=1}^{N} \alpha_{n}(t) z(t,[t]+n)\right|, \quad \text { a.s., a.e. } t \in[0, T], \forall \alpha \in \Gamma(t) .
\end{aligned}
$$

Proof. By (3.16), we have

$$
\begin{aligned}
E[R(T ; \alpha(\cdot))] & =\frac{1}{T} E \int_{0}^{T}\left[r(t)+\Lambda(t, r(t)) h(t, \alpha(t))-\frac{1}{2} h(t, \alpha(t))^{2}\right] d t \\
& =\frac{1}{T} E \int_{0}^{T}\left[r(t)+\frac{1}{2} \Lambda(t, r(t))^{2}-\frac{1}{2}|\Lambda(t, r(t))-h(t, \alpha(t))|^{2}\right] d t .
\end{aligned}
$$


Then

$$
\begin{aligned}
U(T) & =\sup _{\alpha(\cdot) \in \overline{\mathscr{A}}_{\mathrm{ad}}[0, T]} E[R(T ; \alpha(\cdot))] \\
& =\sup _{\alpha(\cdot) \in \overline{\mathscr{A}}_{\mathrm{ad}}[0, T]} E \int_{0}^{T}\left[r(t)+\frac{1}{2} \Lambda(t, r(t))^{2}-\frac{1}{2}|\Lambda(t, r(t))-h(t, \alpha(t))|^{2}\right] d t \\
& =-\inf _{\alpha(\cdot) \in \overline{\mathscr{A}}_{\mathrm{ad}}[0, T]} \frac{1}{2} E \int_{0}^{T}|\Lambda(t, r(t))-h(t, \alpha(t))|^{2} d t+E \int_{0}^{T}\left[r(t)+\frac{1}{2} \Lambda(t, r(t))^{2}\right] d t .
\end{aligned}
$$

Clearly, $\alpha^{*}(\cdot) \in \overline{\mathscr{A}}_{\mathrm{ad}}[0, T]$ is an optimal portfolio process of term-structure management Problem 3.2, only if

$$
E \int_{0}^{T}\left|\Lambda(t, r(t))-h\left(t, \alpha^{*}(t)\right)\right|^{2} d t=\inf _{\alpha(\cdot) \in \overline{\mathscr{A}}_{\mathrm{dd}}[0, T]} E \int_{0}^{T}|\Lambda(t, r(t))-h(t, \alpha(t))|^{2} d t
$$

holds. Since $\alpha(\cdot) \in \overline{\mathscr{A}}_{\mathrm{ad}}[0, T]$, we have

$$
\alpha(t) \in \Gamma(t), \quad \text { a.s., a.e. } t \in[0, T] .
$$

Suppose (3.5), $\alpha^{*}(\cdot)$ is an optimal portfolio process of term-structure management Problem 3.2, if and only if (3.26) holds.

\section{Solution of the term structure management problem}

In this section, we want to solve the term-structure management problem by Theorem 3.4 for some special cases. We assume that

$$
\Gamma:=\left\{\alpha=\left(\alpha_{1}, \alpha_{2}, \ldots, \alpha_{N}\right) \in \mathbb{R}^{N} \mid a_{n} \geq 0, n=1,2, \ldots, N, \sum_{n=1}^{N} \alpha_{n} \leq 1-\alpha_{0}\right\},
$$

where $\alpha_{0}>0$.

For any given $(t, r) \in[0, T] \times \mathbb{R}$, denote

$$
\bar{\Gamma}(t, r)=\left\{\alpha=\left(\alpha_{1}, \alpha_{2}, \ldots, \alpha_{N}\right) \in \Gamma:\left|\sum_{n=1}^{N} \alpha_{n} \frac{\varphi_{r}^{[t]+n}(t, r)}{\varphi^{[t]+n}(t, r)}\right| \leq D_{0}\right\} .
$$

For any $(t, r) \in[0, T] \times \mathbb{R}$, since $0 \in \bar{\Gamma}(t, r), \bar{\Gamma}(t, r) \neq \varnothing$. Moreover, we can check that $\bar{\Gamma}(t, r)$ is a bounded and closed convex set. 
Theorem 4.1. There is a measurable function $\bar{\alpha}(\cdot, \cdot)=\left(\bar{\alpha}_{1}(\cdot, \cdot), \bar{\alpha}_{2}(\cdot, \cdot), \ldots, \bar{\alpha}_{N}(\cdot, \cdot)\right):[0$, $T] \times \mathbb{R} \rightarrow \Gamma$, satisfies

$$
\begin{gathered}
\bar{\alpha}(t, r) \in \bar{\Gamma}(t, r), \quad \forall(t, r) \in[0, T] \times \mathbb{R}, \\
\left|\sum_{n=1}^{N} \bar{\alpha}_{n}(t, r) \zeta(t, r) \frac{\varphi_{r}^{[t]+n}(t, r)}{\varphi^{[t]+n}(t, r)}-\Lambda(t, r)\right| \\
=\inf _{\alpha \in \bar{\Gamma}(t, r)}\left|\sum_{n=1}^{N} \alpha_{n} \zeta(t, r) \frac{\varphi_{r}^{[t]+n}(t, r)}{\varphi^{[t]+n}(t, r)}-\Lambda(t, r)\right|, \quad \text { a.e. }(t, r) \in[0, T] \times \mathbb{R} .
\end{gathered}
$$

Moreover, process $\alpha^{*}(\cdot):=\bar{\alpha}(t, r(t)), t \in[0, T]$, is an optimal bond portfolio process of term structure management Problem 3.2.

Proof. Denote

$$
H^{t, r}(\alpha):=\left|\sum_{n=1}^{N} \alpha_{n} \zeta(t, r) \frac{\varphi_{r}^{[t]+n}(t, r)}{\varphi^{[t]+n}(t, r)}-\Lambda(t, r)\right|, \quad \alpha \in \bar{\Gamma}(t, r)
$$

Let

$$
\begin{gathered}
\bar{H}^{t, r}=\inf _{\alpha \in \bar{\Gamma}(t, r)} H^{t, r}(\alpha), \\
\bar{\Gamma}^{*}(t, r)=\left\{\alpha \in \bar{\Gamma}(t, r) \mid H^{t, r}(\alpha)=\bar{H}^{t, r}\right\} .
\end{gathered}
$$

Since $\bar{\Gamma}(t, r)$ is nonempty, bounded, and closed convex, it is easy to check that $\bar{\Gamma}^{*}(t, r)$ is nonempty. Then by Filippov's lemma, we can find a measurable function $\bar{\alpha}(\cdot, \cdot):[0, T] \times$ $\mathbb{R} \rightarrow \Gamma$, which satisfies $\bar{\alpha}(t, r) \in \bar{\Gamma}^{*}(t, r)$. By the definition, $\bar{\alpha}(\cdot, \cdot)$ satisfies (4.3). Now we denote $\alpha^{*}(t):=\bar{\alpha}(t, r(t)), t \in[0, T]$. It is clear that $\alpha^{*}(\cdot) \in \overline{\mathscr{A}}_{\mathrm{ad}}[0, T]$, which means that, for any $\alpha(\cdot) \in \overline{\mathscr{A}}_{\mathrm{ad}}[0, T]$, note (3.5) and (2.12), we have

$$
\begin{aligned}
& \left|\Lambda(t, r(t))-h\left(t, \alpha^{*}(t)\right)\right| \\
& \quad \leq|\Lambda(t, r(t))-h(t, \alpha(t))|, \quad \text { a.s., a.e. } t \in[0, T], \forall \alpha(\cdot) \in \overline{\mathscr{A}}_{\mathrm{ad}}[0, T] .
\end{aligned}
$$

Consequently, by Theorem 3.4, $\alpha^{*}(\cdot)$ is an optimal bond portfolio process of term-structure management Problem 3.2.

Theorem 4.1 gives a way to find an optimal portfolio process. Denote

$$
\begin{aligned}
G^{+}(t, r) & :=\sup _{\alpha \in \Gamma n=1} \sum_{n=1}^{N} \alpha_{n} \frac{\varphi_{r}^{[t]+n}(t, r)}{\varphi^{[t]+n}(t, r)}, \\
G^{-}(t, r) & :=\inf _{\alpha \in \Gamma n=1}^{N} \sum_{n} \frac{\varphi_{r}^{[t]+n}(t, r)}{\varphi^{[t]+n}(t, r)},
\end{aligned}
$$

and let

$$
D^{+}(t, r):=\min \left\{G^{+}(t, r), D_{0}\right\}, \quad D^{-}(t, r):=\max \left\{G^{-}(t, r),-D_{0}\right\}
$$


Obviously, for any given $D \in\left[D^{-}(t, r), D^{+}(t, r)\right]$, there exists an $\alpha \in \Gamma$ such that

$$
\sum_{n=1}^{N} \alpha_{n} \frac{\varphi_{r}^{[t]+n}(t, r)}{\varphi^{[t]+n}(t, r)}=D
$$

Theorem 4.2. Suppose that

$$
\frac{\Lambda(t, r)}{\zeta(t, r)} \in\left[D^{-}(t, r), D^{+}(t, r)\right], \quad \forall(t, r) \in[0, T] \times \mathbb{R} .
$$

Then $\alpha(\cdot) \in \overline{\mathscr{A}}_{\mathrm{ad}}[0, T]$ is an optimal bond portfolio process of term-structure management Problem 3.2, if and only if

$$
\sum_{n=1}^{N} \alpha_{n}(t) z(t,[t]+n)=\Lambda(t, r(t)), \quad \text { a.s., a.e. } t \in[0, T] .
$$

Proof. By (4.10), for any given $(t, r) \in[0, T] \times \mathbb{R}$, similar to (4.9), there exists an $\alpha=$ $\left(\alpha_{1}, \alpha_{2}, \ldots, \alpha_{N}\right) \in \bar{\Gamma}(t, r)$ such that

$$
\sum_{n=1}^{N} \alpha_{n} \frac{\varphi_{r}^{[t]+n}(t, r)}{\varphi^{[t]+n}(t, r)}=\frac{\Lambda(t, r)}{\zeta(t, r)}
$$

Then there exists at least one function $\bar{\alpha}(\cdot, \cdot):[0, T] \times \mathbb{R} \rightarrow \Gamma$ which satisfies

$$
\begin{gathered}
\bar{\alpha}(t, r) \in \bar{\Gamma}(t, r), \quad \forall(t, r) \in[0, T] \times \mathbb{R}, \\
\sum_{n=1}^{N} \bar{\alpha}_{n}(t, r) \zeta(t, r) \frac{\varphi_{r}^{[t]+n}(t, r)}{\varphi^{[t]+n}(t, r)}=\Lambda(t, r), \quad \forall(t, r) \in[0, T] \times \mathbb{R} .
\end{gathered}
$$

Let $\alpha^{*}(t):=\bar{\alpha}(t, r(t)), t \in[0, T]$. By Theorem 3.4, process $\alpha^{*}(\cdot)$ is an optimal bond portfolio process of term-structure management Problem 3.2.

On the other hand, by the fact that

$$
h\left(t, \alpha^{*}(t)\right)=\Lambda(t, r(t)), \quad \text { a.s., a.e. } t \in[0, T],
$$

and considering (3.28), we have

$$
U(T)=\frac{1}{T} E \int_{0}^{T}\left[r(t)+\frac{1}{2} \Lambda(t, r(t))^{2}\right] d t .
$$

Obviously, $\alpha(\cdot)$ is an optimal bond portfolio process of term-structure management Problem 3.2, only if

$$
E \int_{0}^{T}|\Lambda(t, r(t))-h(t, \alpha(t))|^{2} d t=0
$$

Consequently, $\alpha(\cdot)$ is an optimal bond portfolio process of term-structure management Problem 3.2 if and only if (4.11) holds. 
For the case when assumption (4.10) holds, we can solve Problem 3.2 as the following: first, for $\alpha \in \bar{\Gamma}(t, r)$ solve the following equation:

$$
\sum_{n=1}^{N} \alpha_{n} \zeta(t, r) \frac{\varphi_{r}^{[t]+n}(t, r)}{\varphi^{[t]+n}(t, r)}=\Lambda(t, r)
$$

Denote

$$
\bar{\Gamma}^{*}(t, r):=\left\{\alpha \in \bar{\Gamma}(t, r): \sum_{n=1}^{N} \alpha_{n} \zeta(t, r) \frac{\varphi_{r}^{[t]+n}(t, r)}{\varphi^{[t]+n}(t, r)}=\Lambda(t, r)\right\}
$$

to be the solution set of (4.17). Next, construct a measurable function $\bar{\alpha}(\cdot, \cdot):[0, T] \times$ $\mathbb{R} \rightarrow \Gamma$ which satisfies

$$
\bar{\alpha}(t, r) \in \bar{\Gamma}^{*}(t, r), \quad \forall(t, r) \in[0, T] \times \mathbb{R} .
$$

Finally, define a process $\alpha^{*}(\cdot): \alpha^{*}(t)=\bar{\alpha}(t, r(t)), t \in[0, T]$, which is an optimal bond portfolio process of term-structure management Problem 3.2.

If assumption (4.10) does not hold, we can also construct an optimal bond portfolio process. Denote

$$
\begin{gathered}
\mathcal{O}^{+}:=\left\{(t, r) \in[0, T] \times \mathbb{R} \mid \frac{\Lambda(t, r)}{\zeta(t, r)} \geq D^{+}(t, r)\right\}, \\
\mathcal{O}^{*}:=\left\{(t, r) \in[0, T] \times \mathbb{R} \mid \frac{\Lambda(t, r)}{\zeta(t, r)} \in\left[D^{-}(t, r), D^{+}(t, r)\right]\right\}, \\
\mathcal{O}^{-}:=\left\{(t, r) \in[0, T] \times \mathbb{R} \mid \frac{\Lambda(t, r)}{\zeta(t, r)} \leq D^{-}(t, r)\right\} .
\end{gathered}
$$

By the definition, if $(t, r) \in \mathbb{O}^{*}$, we have (4.10) holds. If $(t, r) \in \mathbb{O}^{+}$, let

$$
\bar{\Gamma}^{+}(t, r):=\left\{\alpha \in \Gamma: \sum_{n=1}^{N} \alpha_{n} \frac{\varphi_{r}^{[t]+n}(t, r)}{\varphi^{[t]+n}(t, r)}=D^{+}(t, r)\right\}
$$

and if $(t, r) \in 0^{-}$, let

$$
\bar{\Gamma}^{-}(t, r):=\left\{\alpha \in \Gamma: \sum_{n=1}^{N} \alpha_{n} \frac{\varphi_{r}^{[t]+n}(t, r)}{\varphi^{[t]+n}(t, r)}=D^{-}(t, r)\right\} .
$$

Then by Filippov's lemma, there is a measurable function $\bar{\alpha}^{*}(\cdot, \cdot):[0, T] \times \mathbb{R} \rightarrow \Gamma$ which satisfies
(I) $\bar{\alpha}^{*}(t, r) \in \bar{\Gamma}^{+}(t, r)$, if $(t, r) \in \mathrm{O}^{+}$,
(II) $\bar{\alpha}^{*}(t, r) \in \bar{\Gamma}^{*}(t, r)$, if $(t, r) \in \mathbb{O}^{*}$,
(III) $\bar{\alpha}^{*}(t, r) \in \bar{\Gamma}^{-}(t, r)$, if $(t, r) \in 0^{-}$.

For the measurable function $\bar{\alpha}^{*}(\cdot, \cdot)$ constructed above, we have the following result. 
Theorem 4.3. The process $\alpha^{*}(\cdot):=\bar{\alpha}^{*}(t, r(t)), t \in[0, T]$, is an optimal bond portfolio process of term-structure management Problem 3.2.

Proof. It is easy to check that $\bar{\alpha}^{*}(t, r)$ satisfies conditions (4.3). Then by Theorem 4.1, Theorem 4.3 is clear.

\section{Conclusion}

In the definition of traditional duration, it needs to assume that the yield curves are flat and shift in a parallel manner, it is very uncomfortable for practical applications. In this paper, we introduced a new definition of duration of bond portfolios under stochastic interest rate model. This provides a more accurate measurement for the interest rate risk than the traditional duration. Then we considered an optimal bond investment termstructure management problem using this duration as a performance index. Under some technique conditions, the optimal bond portfolio process is obtained.

\section{References}

[1] Basel Committee on Banking Supervision, Principles for the Management and Supervision of Interest Rate Risk, Supporting Document to the New Basel Capital Accord (2001), Bank for International Settlements.

[2] G. O. Bierwag and G. G. Kaufman, Duration gap for financial institutions, Financial Analysts Journal 41 (1985), no. 2, 68-71.

[3] G. O. Bierwag, G. G. Kaufman, and A. Toevs, Duration: its development and use in bond portfolio management, Financial Analysts Journal 39 (1983), no. 4, 15-35.

[4] T. Björk, Interest rate theory, Financial Mathematics (Bressanone, 1996) (W. J. Runggaldier, ed.), Lecture Notes in Math., vol. 1656, Springer, Berlin, 1997, pp. 53-122.

[5] F. Black, E. Derman, and W. Toy, A one-factor model of interest rates and its application to treasury bond options, Financial Analysts Journal 46 (1990), no. 1, 33-39.

[6] D. Brigo and F. Mercurio, Interest Rate Models—Theory and Practice, Springer Finance, Springer, Berlin, 2001.

[7] J. Y. Campbell, A. W. Lo, and A. C. MacKinlay, The Econometrics of Financial Markets, Princeton University Press, New Jersay, 1997.

[8] D. M. Chance, Default risk and the duration of zero-coupon bonds, Journal of Finance 45 (1990), no. $1,265-274$.

[9] A. G. Cornyn, R. A. Klein, and J. Lederman, Controlling and Managing Interest-Rate Risk, NYIF, New York, 1997.

[10] J. C. Cox, J. E. Ingersoll Jr., and S. A. Ross, A theory of the term structure of interest rates, Econometrica 53 (1985), no. 2, 385-407.

[11] N. El Karoui, S. G. Peng, and M. C. Quenez, Backward stochastic differential equations in finance, Mathematical Finance. An International Journal of Mathematics, Statistics and Financial Economics 7 (1997), no. 1, 1-71.

[12] W. B. English, Interest rate risk and bank net interest margins, BIS Quarterly Review, December (2002), 67-82.

[13] F. Fabozzi, Bond Markets, Analysis and Strategies, 4th ed., Prentice-Hall, New Jersay, 2000.

[14] T. S. Y. Ho, Key rate durations: a measure of interest-rate risk, Journal of Fixed Income 2 (1992), no. 2, 29-44.

[15] J. E. Ingersoll Jr., J. Skelton, and R. Weil, Duration forty years later, Journal of Financial and Quantitative Analysis 13 (1978), no. 4, 627-650. 
[16] J. Ma, P. Protter, and J. M. Yong, Solving forward-backward stochastic differential equations explicitly — a four step scheme, Probability Theory and Related Fields 98 (1994), no. 3, 339-359.

[17] J. Ma and J. M. Yong, Forward-Backward Stochastic Differential Equations and Their Applications, Lecture Notes in Mathematics, vol. 1702, Springer, Berlin, 1999.

[18] É. Pardoux and S. G. Peng, Adapted solution of a backward stochastic differential equation, Systems \& Control Letters 14 (1990), no. 1, 55-61.

[19] M. Rutkowski, Self-financing trading strategies for sliding, rolling-horizon, and consol bonds, Mathematical Finance. An International Journal of Mathematics, Statistics and Financial Economics 9 (1999), no. 4, 361-385.

[20] S. Sarkar and G. Hong, Effective duration of callable corporate bonds: theory and evidence, Journal of Banking \& Finance 28 (2004), no. 3, 499-521.

[21] G. M. Soto, Duration models and IRR management: a question of dimensions?, Journal of Banking \& Finance 28 (2004), no. 5, 1089-1110.

[22] L. Thomas and R. Willner, Measuring the duration of an international diversified bond portfolio, Journal of Portfolio Management, fall (1997), 93-97.

[23] A. L. Toevs, Gap management: managing interest rate risk in banks and thrifts, Federal Reserve Bank of San Francisco: Economic Review 2 (1983), 20-35.

Daobai Liu: Institute for Financial Studies, Fudan University, Shanghai 200433, China

E-mail address: dbliu@fudan.edu.cn 


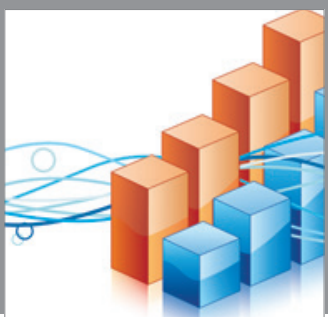

Advances in

Operations Research

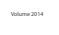

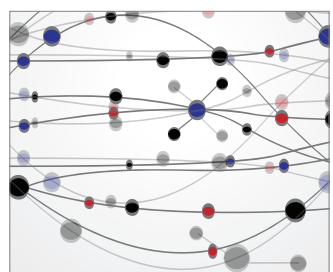

\section{The Scientific} World Journal
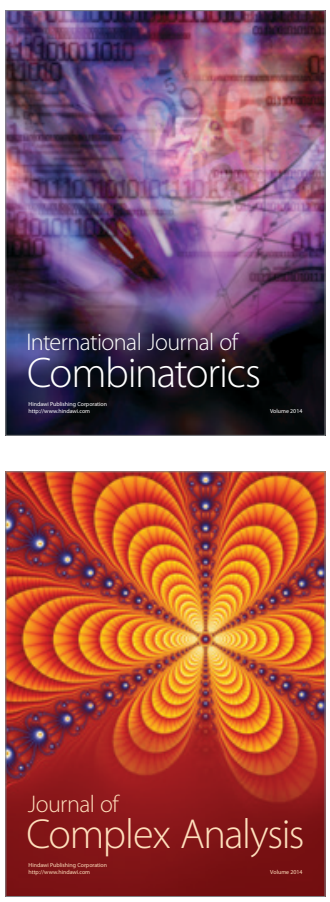

International Journal of

Mathematics and

Mathematical

Sciences
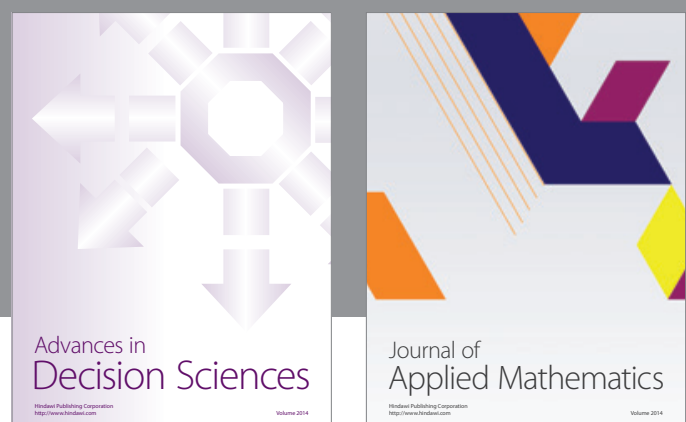

Journal of

Applied Mathematics
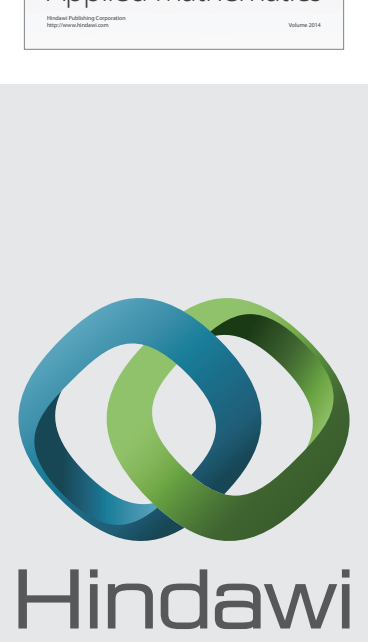

Submit your manuscripts at http://www.hindawi.com
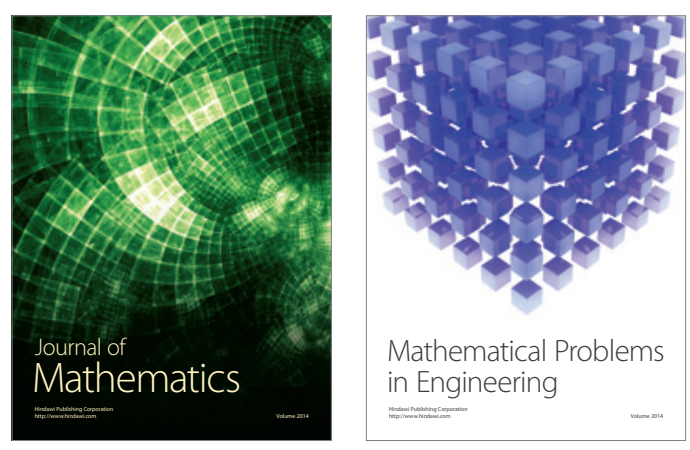

Mathematical Problems in Engineering
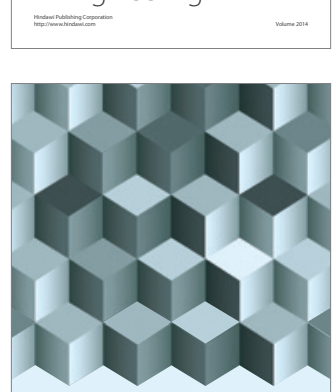

Journal of

Function Spaces
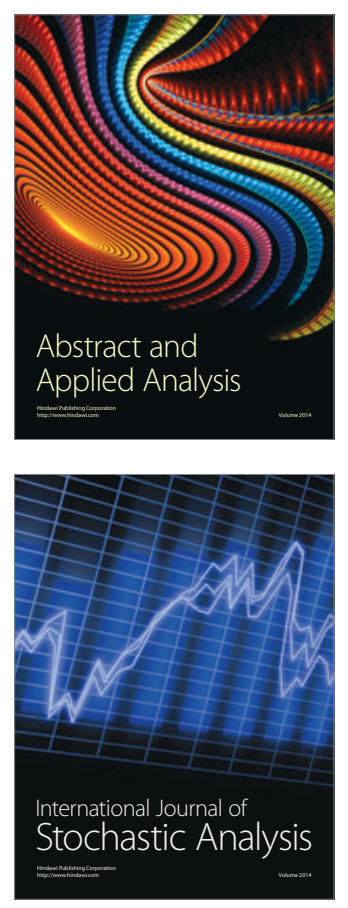

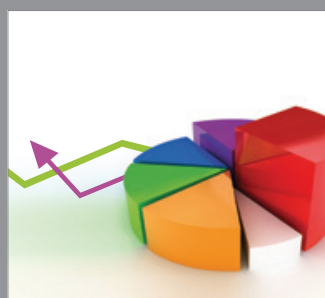

ournal of

Probability and Statistics

Promensencen
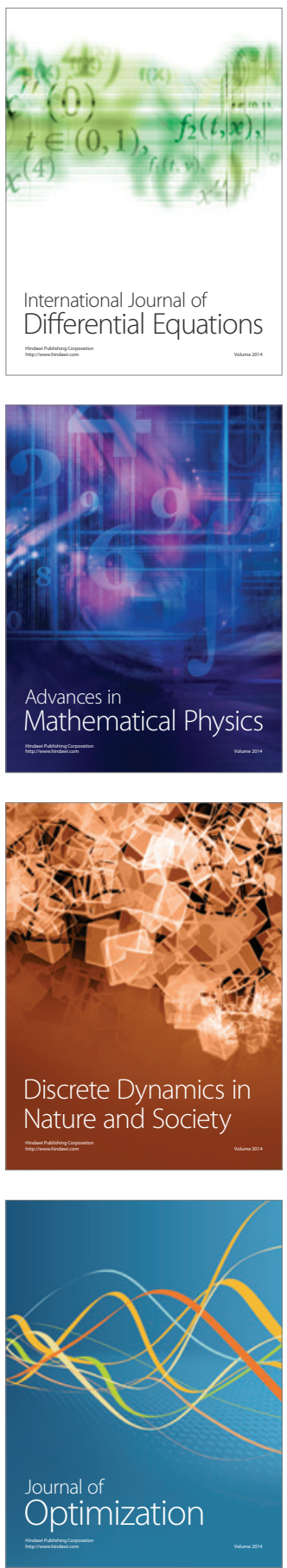